\title{
Comparative analysis of canine mesenchymal stem cells and bone marrow-derived mononuclear cells
}

\author{
Noritaka Maeta ${ }^{1,2}(\mathbb{D})$, Katsutoshi Tamura ${ }^{1}\left(\mathbb{D}\right.$, Fuuna Ezuka ${ }^{3}(\mathbb{D})$ and Hiroshi Takemitsu ${ }^{3,4}(\mathbb{D})$
}

1. Aikouishida Animal Hospital, Isehara, 1195-4 Takamori, Isehara, Kanagawa, 259-1114, Japan; 2. Faculty of Veterinary Medicine, Okayama University of Science, 1-3 Ikoinooka, Imabari, Ehime, 794-8555, Japan; 3. Science and Humanities Master's Programme, Graduate School of Science and the Humanities, Kurashiki University of Science and The Arts, 2640 Nishinoura Tsurajima Kurashiki Okayama, 712-8505, Japan; 4. Department of Comparative Animal Science, College of Life Science, Kurashiki University of Science and The Arts, 2640 Nishinoura Tsurajima Kurashiki Okayama, 712-8505, Japan.

Corresponding author: Hiroshi Takemitsu, e-mail: takemitsu@kusa.ac.jp

Co-authors: NM: n-maeta@vet.ous.ac.jp, KT: katsutoshi@vet.name, FE: h22hm02@kusa.ac.jp, Received: 27-10-2020, Accepted: 16-03-2021, Published online: 29-04-2021

doi: www.doi.org/10.14202/vetworld.2021.1028-1037 How to cite this article: Maeta N, Tamura K, Ezuka F, Takemitsu H (2021) Comparative analysis of canine mesenchymal stem cells and bone marrow-derived mononuclear cells, Veterinary World, 14(4): 1028-1037.

\begin{abstract}
Background and aim: Mesenchymal stem cells (MSCs), which have multi-lineage differentiation potentials, are a promising source for regenerative medicine. However, the focus of study of MSCs is shifting from the characterization of the differentiation potential to their secretion potential for cell transplantation. Tissue regeneration and the attenuation of immune responses are thought to be affected by the secretion of multiple growth factors and cytokines by MSCs. However, the secretion potential of MSCs profiling remains incompletely characterized. In this study, we focused on the secretion ability related and protein mRNA expression of dog adipose tissue-derived MSCs (AT-MSC), bone marrow (BM)-derived MSCs, and BM-derived mononuclear cells (BM-MNC).

Materials and Methods: Real-time polymerase chain reaction analyses revealed mRNA expression of nine growth factors and seven interleukins in these types of cells and three growth factors protein expression were determined using Enzymelinked immunosorbent assay.

Results: For the BM-MNC growth factors, the mRNA expression of transforming growth factor- $\beta$ (TGF- $\beta$ ) was the highest. For the BM-derived MSC (BM-MSC) and AT-MSC growth factors, the mRNA expression of vascular endothelial growth factor (VEGF) was highest. BM-MSCs and AT-MSCs showed similar expression profiles. In contrast, BM-MNCs showed unique expression profiles for hepatocyte growth factor and epidermal growth factor. The three types of cells showed a similar expression of TGF- $\beta$.
\end{abstract}

Conclusion: We conclude that expression of cytokine proteins and mRNAs suggests involvement in tissue repair and protection.

Keywords: bone marrow-derived mononuclear cell, canine, growth factor, interleukin, mesenchymal stem cell, secretion.

\section{Introduction}

Mesenchymal stem cells (MSCs) are known to have multi-lineage differentiation potential, ability for rapid proliferation, and capacity for self-renewal [1-3]. In addition, they are widely present in tissues, such as bone marrow (BM), adipose tissue (AT), umbilical cord, and muscle [4-6]. MSCs can differentiate into various tissues in the appropriate microenvironment [7]. MSCs hold promise for regenerative medicine due to their accessibility, expandability, and multipotentiality. Recent research also finds interest in MSCs' secretion potential, as well as their differentiation, proliferation, and self-renewal potentials [8]. Moreover, studies reveal that MSCs secrete various

Copyright: Maeta, et al. Open Access. This article is distributed under the terms of the Creative Commons Attribution 4.0 International License (http://creativecommons.org/licenses/by/4.0/), which permits unrestricted use, distribution, and reproduction in any medium, provided you give appropriate credit to the original author(s) and the source, provide a link to the Creative Commons license, and indicate if changes were made. The Creative Commons Public Domain Dedication waiver (http://creativecommons.org/ publicdomain/zero/1.0/) applies to the data made available in this article, unless otherwise stated. cytokines, such as epidermal growth factor (EGF), fibroblast growth factor (FGF), and vascular endothelial growth factor (VEGF) [9,10]. These cytokines are thought to work synergistically with the differentiation potential of MSCs in tissues repair [11,12]. Significant research also reveals the potential of MSCs to secrete immunosuppressive cytokines and their involvement in preventing acute inflammation in tissues, such as myocardium, nerves, and dacryoadenitis [12-14]. It has been suggested that these effects are caused by cytokines.

BM-MNCs have been used to treat leukemia by cell transplantation as this method is very safe [15]. Since BM-MNCs consist of MSCs, hematopoietic stem cells, fibroblasts, and other cells, they have been conjectured to have a secretion potential which is different from that of BM-MSCs. BM-MNCs are also known to be mobilized by response to the release of inflammatory cytokines in the peripheral blood by injured tissues; these cells eventually reach injured tissue and contribute to their regeneration [16]. This behavior is evident in several tissues in response 
to injuries, such as acute myocardial infarction, hepatic failure, and renal failure [15,17]. Moreover, we have recently performed a clinical trial that used BM-MNCs in a dog with an acute spinal cord injury resulting from intervertebral disc herniation; results showed that BM-MNC transplantation resulted in a significant increase in the recovery rate of spinal cord injury [17]. Hence, the secretion of growth factors and cytokines by BM-MNCs allowed for these recoveries.

However, there have been few studies on the secretion potential of canine BM-MNCs. In general, growth factors are proteins that promote growth and proliferation of particular cells. Brain-derived neurotrophic factor (BDNF), nerve growth factor (NGF), and neurotrophin 3 were involved in protection, proliferation, or differentiation of nerve $[18,19]$. EGF, FGF-2a, and hepatocyte grows factor (HGF) are related to the proliferation of many cells [20-22]. Platelet-derived growth factor-C (PDGF-C) and VEGF-A play a crucial role in angiogenesis [23] and transforming growth factor (TGF- $\beta 1$ ) is an inducer of fibroblasts [24]. These capabilities are a function indispensable for tissue repair. Interleukin (IL) is a cytokine related to inflammation and (IL)-1a, IL-1b, IL-6, IL-11, and IL-17A are well known to promote inflammation [25-27]. However, IL-4 and IL-10 function differently for these ILs suppress inflammation [28]. Few studies have been conducted on these cytokines in an attempt to measure them simultaneously in the MSCs of the dog. Here, we evaluated the mRNA expression profile of nine growth factors, seven ILs, and TNF- $\alpha$ in canine BM-MSCs, AT-MSCs, and BM-MNCs by quantitative real-time polymerase chain reaction (qPCR). In addition, we examined the protein expression of VEGF-A, HGF, and TGF- $\beta 1$.

This study was aimed at characterizing the secretion profile of canine MSCs and MNCs isolated from BM and AT. Our study is able to provide important findings for the basic research of cell transplantation therapy.

\section{Materials and Methods}

\section{Ethical approval}

Animal experiments were carried out in accordance with the National Institutes of Health guidelines for the care and use of laboratory animals. This study and the protocol included herewith were approved by the Aikouishida Animal Hospital Committee for Animal Experimentation.

\section{Study period and location}

The study was conducted from April 2019 to November 2019 in Aikouishida Animal Hospital and Kurashiki University of Science and The Arts.

\section{Animals}

The study included three young healthy female beagle dogs (1 year old, 9.5-11.3 kg body weight).
Before tissue sample isolation, anesthesia with propofol (Hospira, Osaka, Japan) $(7 \mathrm{mg} / \mathrm{kg})$ by intravenous injection was applied. After intubation, anesthesia was maintained with isoflurane (1.5-2.0\%) in oxygen.

\section{Cell isolation and culture}

BM was aspirated from the proximal humerus with a general BM biopsy technique under anesthesia. In brief, a sterilized 13-gauge Jamshidi needle (Cardinal Health, McGaw Park, IL, USA) was used to aspirate $5 \mathrm{~mL}$ of $\mathrm{BM}$ into a syringe containing $5 \mathrm{~mL}$ of heparinized (1000 units $/ \mathrm{mL}$ ) saline solution. Ten milliliters of $\mathrm{BM} /$ saline mixture was carefully layered on $5 \mathrm{~mL}$ of density gradient medium (density, 1.077; Lymphoprep; Nycomed Pharma, Oslo, Norway) and then centrifuged at $500 \times \mathrm{g}$ for $30 \mathrm{~min}$. The suspended cloud-like layer of BM-MNCs was carefully collected, washed twice in $10 \mathrm{~mL}$ of physiological saline and centrifuged at $300 \times \mathrm{g}$ for $5 \mathrm{~min}$. These cells were used for the qPCR assay, and the remaining cells were separated into 6-well plates and $75-\mathrm{cm}^{2}$ cell-culture flasks to separate BM-MNCs and BM-MSCs. Each cell was then resuspended in Dulbecco's modified Eagle's medium (Invitrogen, Carlsbad, CA, USA) with 10\% fetal bovine serum (HyClone Laboratories, Utah, USA) and a 1\% antibiotic-antimycotic solution (Invitrogen) and incubated at $37^{\circ} \mathrm{C}$ in a humidified incubator with $5 \% \mathrm{CO}_{2}$. The 6-well-plate-seeded cells were plated at a density of $5.0 \times 10^{3} \mathrm{cells} / \mathrm{cm}^{2}$ and the conditioned medium for ELISA was collected once every 2 days.

The $75-\mathrm{cm}^{2}$-cell-culture-flask-seeded cells were cultured until $70-80 \%$ confluency, and then the attached cells were passaged by exposure to $0.25 \%$ trypsin for $3 \mathrm{~min}$ and replated at a density of $8.0 \times 10^{3}$ cells $/ \mathrm{cm}^{2}$ for subsequent passage. Passage 2 cells were separated into 6-well plates at a density of $5.0 \times 10^{3} \mathrm{cells} / \mathrm{cm}^{2}$ for ELISA and $75-\mathrm{cm}^{2}$ cell-culture flasks for qPCR.

AT was also harvested from each dog under general anesthesia. Subcutaneous fat pads (approximately $1.0 \mathrm{~g})$ were harvested from the inguinal area. These pads were finely minced with scissors, digested in $40 \mathrm{~mL}$ of phosphate-buffered saline (PBS) containing $0.15 \%$ type- 1 collagenase (Sigma-Aldrich, St. Louis, $\mathrm{MO}$, USA), and then shaken vigorously at $37^{\circ} \mathrm{C}$ for $60 \mathrm{~min}$. Subsequently, the samples were then filtered using $100 \mu \mathrm{m}$ cell strainers (BD Biosciences, Franklin Lakes, USA) and washed with PBS. The samples were then filtered with $100-\mu \mathrm{m}$ cell strainers (BD Biosciences,) and washed with PBS. The obtained cells were seeded into $75-\mathrm{cm}^{2}$ cell-culture flasks with $10 \mathrm{~mL}$ of control medium and incubated in the same manner as the BM cells.

\section{Reverse transcription and qPCR}

Total RNA was obtained from cultured BM-MSCs and AT-MSCs in passage 1. Total RNA was extracted with a TRIzol reagent (Invitrogen) according to the manufacturer's protocol. Total RNA was measured 
by spectrophotometry. Total RNA $(1 \mu \mathrm{g})$ was reverse transcribed at $42^{\circ} \mathrm{C}$ for $15 \mathrm{~min}$ in $20 \mu \mathrm{L}$ of QuantiTect (Qiagen, Düsseldorf, Germany) after inactivation of reverse transcription by heating at $95^{\circ} \mathrm{C}$ for $3 \mathrm{~min}$.

Previously, we conducted experiments on and mRNA expression in cultured cells [29]. The cDNA product was subjected to qPCR according to the user instructions for the Real-Time PCR System 7300 (Applied Biosystems, Foster City, CA, USA). qPCR was performed at $95^{\circ} \mathrm{C}$ for $5 \mathrm{~s}$ and $60^{\circ} \mathrm{C}$ for $34 \mathrm{~s}$ in $20 \mu \mathrm{L}$ of buffer containing SYBR premix ExTaq II and ROX Reference Dye (Takara Bio, Shiga, Japan) and $0.2 \mu \mathrm{M}$ each of the primers (Tables-1 and 2). Each primer was designed based on information on GenBank. The value of mRNA expression was calculated and expressed as copies (copies/ng of cDNA input) or normalized to beta-actin. Each primer is used after confirming that the PCR product has the correct sequence. A linear amplification curve from serial dilutions of plasmid DNA containing each cDNA was established through quantitative measurements. Each reaction was performed in triplicate.

\section{Enzyme-linked immunosorbent assay (ELISA)}

Previously, we conducted experiments on protein expression in cultured cells [29]. The samples used for the experiments were obtained from BM-MNC, BM-MSC, and Adipose-derived-MSC (AD-MSC) culture-conditioned medium and collected and frozen at $-80^{\circ} \mathrm{C}$ until the assay. HGF (Hepatocyte growth factor ELISA kit; USCN, Huston USA), VEGF-A (Quantikine ELISA canine VEGF; R\&D Systems, Inc., Minneapolis, MN, USA), and TGF- $\beta 1$ (Quantikine ELISA Mouse/Rat/Porcine/Canine TGF$\beta 1$; R\&D Systems, Inc.) were determined according to the manufacturer's protocol. D-MEM containing $10 \%$ FBS was used as a blank. All ELISA kits were guaranteed by the manufacturer for use in dogs.

Table-1: Primers used for qPCR.

\begin{tabular}{|c|c|c|c|c|}
\hline Name & $5^{\prime}-3^{\prime}$ & Direction & Position & GenBank No. \\
\hline Bdnf-1 & AATCCCATGGGTTACACGAA & sense & 581 & NM_001002975.1 \\
\hline Bdnf-2 & GCCAGCCAATTCTCTITTG & anti-sense & 707 & NM_001002975.1 \\
\hline Egf-1 & GCTGTGTCATTGGATGTGCT & sense & 616 & NM_001003094.1 \\
\hline Egf-2 & CTGATTCCCAAAAAGGGACAT & anti-sense & 780 & NM_001003094.1 \\
\hline Fgf-1 & CACTTCAAGGACCCCAAGAG & sense & 190 & XM_003432481.1 \\
\hline Fgf-2 & ACAACGCCTCTCTCTTCTGC & anti-sense & 332 & XM_003432481.1 \\
\hline $\mathrm{Hgf}-1$ & ATGGGGAATGAGAAATGCAG & sense & 1915 & $A B 090353.1$ \\
\hline $\mathrm{Hgf}-2$ & AAAAATGCCAGGACGATTTG & anti-sense & 2124 & AB090353.1 \\
\hline Ngf-1 & GTGCTGGGAGAGGTGAACAT & sense & 475 & NM_001194950.1 \\
\hline $\mathrm{Ngf}-2$ & GGTGGTGGTGCAGTAGGAGT & anti-sense & 612 & NM_001194950.1 \\
\hline Nt3-1 & TGGCATCCAAGGTAACAACA & sense & 311 & XM_003433475.1 \\
\hline $\mathrm{Nt3}-2$ & GCAGGGTGCTCTGGTAGTTC & anti-sense & 459 & XM_003433475.1 \\
\hline Pdgf-1 & TCTTGGCAAGGCTITGTT & sense & 849 & XM_539783.3 \\
\hline Pdgf-2 & TTCCСTTATGGACACCGAGA & anti-sense & 966 & XM_539783.3 \\
\hline Tgf-1 & GGCCCTGGACACCAACTACT & sense & 891 & NM_001003309.1 \\
\hline Tgf-2 & GCTCATGGATCCACTTCCAG & anti-sense & 997 & NM_001003309.1 \\
\hline Vegf-1 & CTACCTCCACCATGCCAAGT & sense & 325 & NM_001003175.2 \\
\hline Vegf-2 & AGATGTCCACCAGGGTCTCA & anti-sense & 458 & NM_001003175.2 \\
\hline
\end{tabular}

$\mathrm{qPCR}=\mathrm{Quantitative} \mathrm{real-time} \mathrm{polymerase} \mathrm{chain} \mathrm{reaction}$

Table-2: Primers used for qPCR.

\begin{tabular}{|c|c|c|c|c|}
\hline Name & $5^{\prime}-3^{\prime}$ & Direction & Position & GenBank No. \\
\hline IL-1a-1 & TTGTGAGTGCCCAAAATGAA & sense & 648 & NM_001003157.2 \\
\hline IL-1a-2 & CCTGTGTGGCAATGAACAAC & anti-sense & 812 & NM_001003157.2 \\
\hline IL-1b-1 & AGTTGCAAGTCTCCCACCAG & sense & 149 & NM_001037971.1 \\
\hline IL-1b-2 & TATCCGCATCTGTITTGCAG & anti-sense & 325 & NM_001037971.1 \\
\hline IL-4-1 & СТСАССТСССААСТGАТТСС & sense & 70 & NM_001003159.1 \\
\hline IL-4-2 & AGTCGTTTCTCGCTGTGAGG & anti-sense & 202 & NM_001003159.1 \\
\hline IL-6-1 & GGCTACTGCTITCССТАССС & sense & 108 & U12234.1 \\
\hline IL-6-2 & गाTCTGCCAGTGCСTCTा & anti-sense & 305 & U12234.1 \\
\hline IL-10-1 & AGAACCACGACCCAGACATC & sense & 300 & U33843.1 \\
\hline IL-10-2 & CCGCCTTGСTCTTATTCTCA & anti-sense & 425 & U33843.1 \\
\hline IL-11-1 & CGGCTGGAAATTTGTCTCTC & sense & 251 & XM_848962.2 \\
\hline IL-11-2 & GGCCAGATAGAGCTGCTG & anti-sense & 456 & XM_848962.2 \\
\hline IL-17-1 & CCGATCTACСTCACCTTGGA & sense & 214 & NM_001165878.1 \\
\hline IL-17-2 & TCGCAGAACCAGGATCTCTT & anti-sense & 379 & NM_001165878.1 \\
\hline Tnf-1 & АССАСАСТСТTСТGССТGСТ & sense & 133 & NM_001003244.4 \\
\hline Tnf-2 & CTTGGGGTTCGAGAAGATGA & anti-sense & 254 & NM_001003244.4 \\
\hline$\beta$-actin & GCCAACCGTGAGAAGATGACT & sense & 339 & AF021873 \\
\hline$\beta$-actin & CCCAGAGTCCATGACAATACCAG & anti-sense & 446 & AF021873 \\
\hline
\end{tabular}

qPCR $=$ Quantitative real-time polymerase chain reaction 


\section{Statistical analysis}

Experimental values are expressed as mean \pm standard error of the mean. A Holm-Sidak 1-way analysis of variance (ANOVA) was utilized to perform multiple group comparisons. Statistical significance was set at $\mathrm{p}<0.05$ for both the U-test and the one-way ANOVA. The statistical analyses were performed using Prism software (GraphPad Software Inc., San Diego, CA, USA).

\section{Results}

Growth factor mRNA expression profiles in BM-MNCs, BM-MSCs, and AT-MSCs

Total RNA was obtained from cultured BM-MSCs and AT-MSCs in passage 2. BM-MNCs were obtained by isolation from BM immediately after separation with Lymphoprep. qPCR was used to examine the expression levels of the growth factors in BM-MNCs, BM-MSCs, and AD-MSCs (Figure-1). For the BM-MNC growth factors, the expression of TGF- $\beta 1$ was highest. A moderate level of expression in BM-MNCs was observed for VEGF-A, HGF, and EGF. Furthermore, a low level of expression in BM-MNCs was observed for PDGF-C, BDNF, FGF-2a, and NGF (Figure-1a).

For the BM-MSC growth factors, the expression of VEGF-A was highest. A moderate level of expression in BM-MSCs was observed for TGF- $\beta 1$, PDGF-C, NGF, and FGF-2a. Moreover, a low level of expression in BM-MSCs was observed for HGF, BDNF, EGF, and NT-3 (Figure-1b).

For the AT-MSC growth factors, the expression of VEGF-A was highest. A moderate level of expression in AT-MSCs was observed for TGF- $\beta 1$, PDGF-C, NGF, FGF-2a, and BDNF. Moreover, a low level of expression in AT-MSCs was observed for EGF, HGF, and NT-3. Each value of mRNA expression was calculated and expressed as copies (Figure-1c).

ILs and TNF- $\alpha$ mRNA expression profiles in BM-MNCs, BM-MSCs, and AT-MSCs

For the ILs and TNF- $\alpha$ in BM-MNCs, the expression of IL-1 $\mathrm{b}$ was highest. A moderate level of expression in BM-MNCs was observed for TNF-a, IL-10, and IL-4. A low level of expression in BM-MNCs was observed for IL-17A, IL-1a, IL-11, and IL-6 (Figure-1d). For the ILs and TNF- $\alpha$ in BM-MSCs, the expression of IL-11 was highest. A moderate level of expression in BM-MSCs was observed for IL-6. A low level of expression in BM-MSCs was observed for IL-1b, IL-17A, TNF-a, IL-10, IL-4, and IL-1a (Figure-1e). For the ILs and TNF- $\alpha$ in AT-MSCs, the expression of IL-11 was highest. A low level of expression in AT-MSCs was observed for

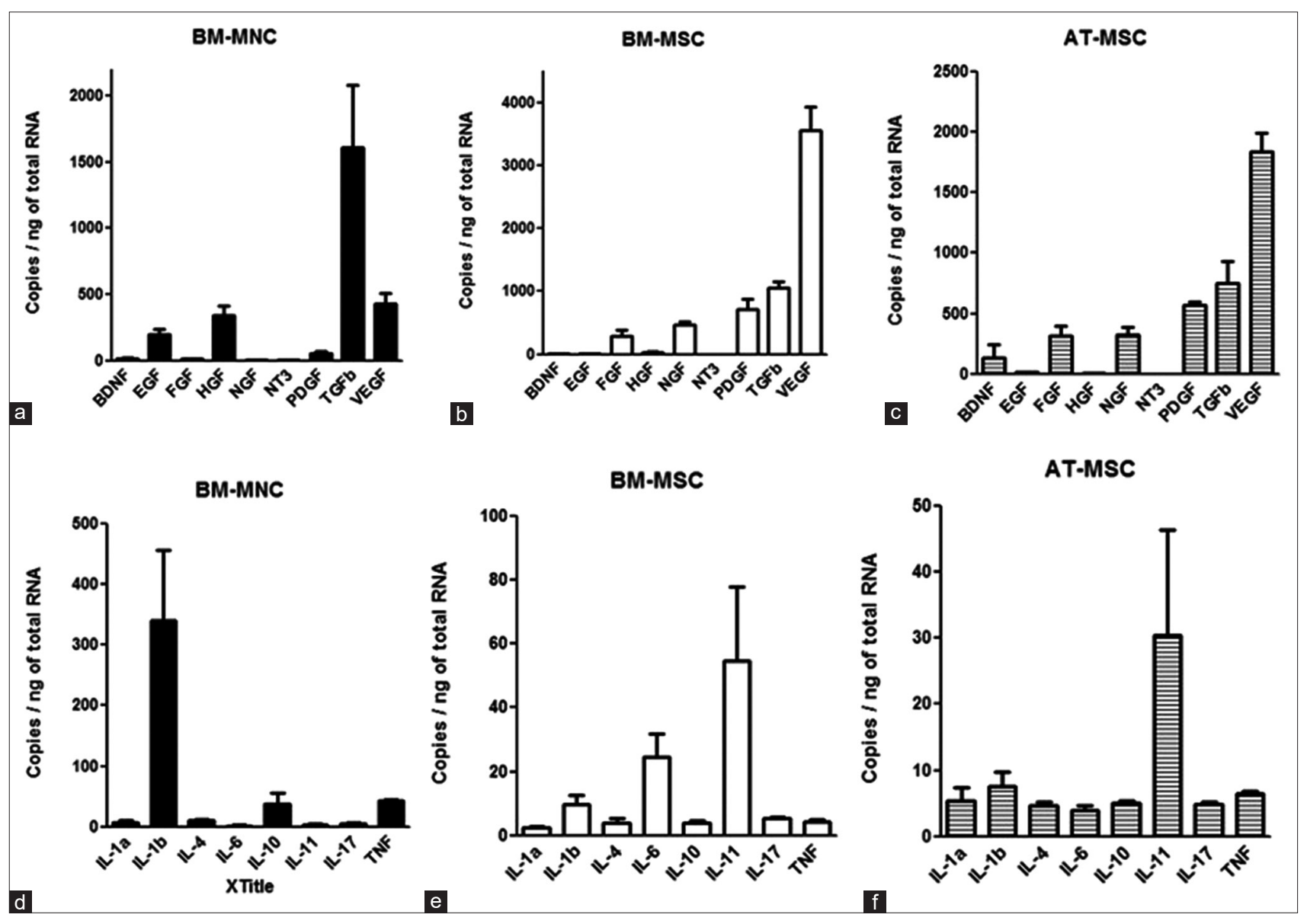

Figure-1: Quantitative real-time polymerase chain reaction. The levels of expression of the dog cytokine mRNAs in bone marrow-derived mononuclear cells, adipose tissue-derived mesenchymal stem cells, and bone marrow-derived mesenchymal stem cells. Each value of mRNA expression was calculated and expressed as copies (copies/ng of cDNA input). Means $\pm S E, n=3$. 
IL-1b, TNF- $\alpha$, IL-1a, IL-10, IL-17A, IL-4, and IL-6 (Figure-1f). Each value of mRNA expression was calculated and expressed as copies (copies/ng of total RNA).

\section{Growth factor mRNA expression}

The mRNA expression of BM-MSC and AT-MSC growth factors tended to be similar, with the exception of a few growth factors, such as BDNF, PDGF-C, and VEGF-A (Figure-2 and Table-3). However, a significant difference between BM-MNC expression compared with BM-MSCs and AT-MSCs was observed.
EGF expression in BM-MNCs was 22.8 fold and 23.9 fold higher than that of BM-MSCs and AT-MSCs, respectively, and $\mathrm{HGF}$ expression was 8.0 fold and 87.0 fold higher, respectively. Conversely, FGF-2a, NGF, PDGF-C, and VEGF-A mRNA expressions in BM-MSCs and AT-MSCs were higher than those of BM-MNCs. FGF-2a expression in BM-MSCs and AT-MSCs was 22.8 fold and 23.9 fold higher, respectively, than that in BM-MNCs and NGF expression in BM-MSCs and AT-MSCs was 240.0 fold higher than that in BM-MNCs. PDGF-C expression in BM-MSCs

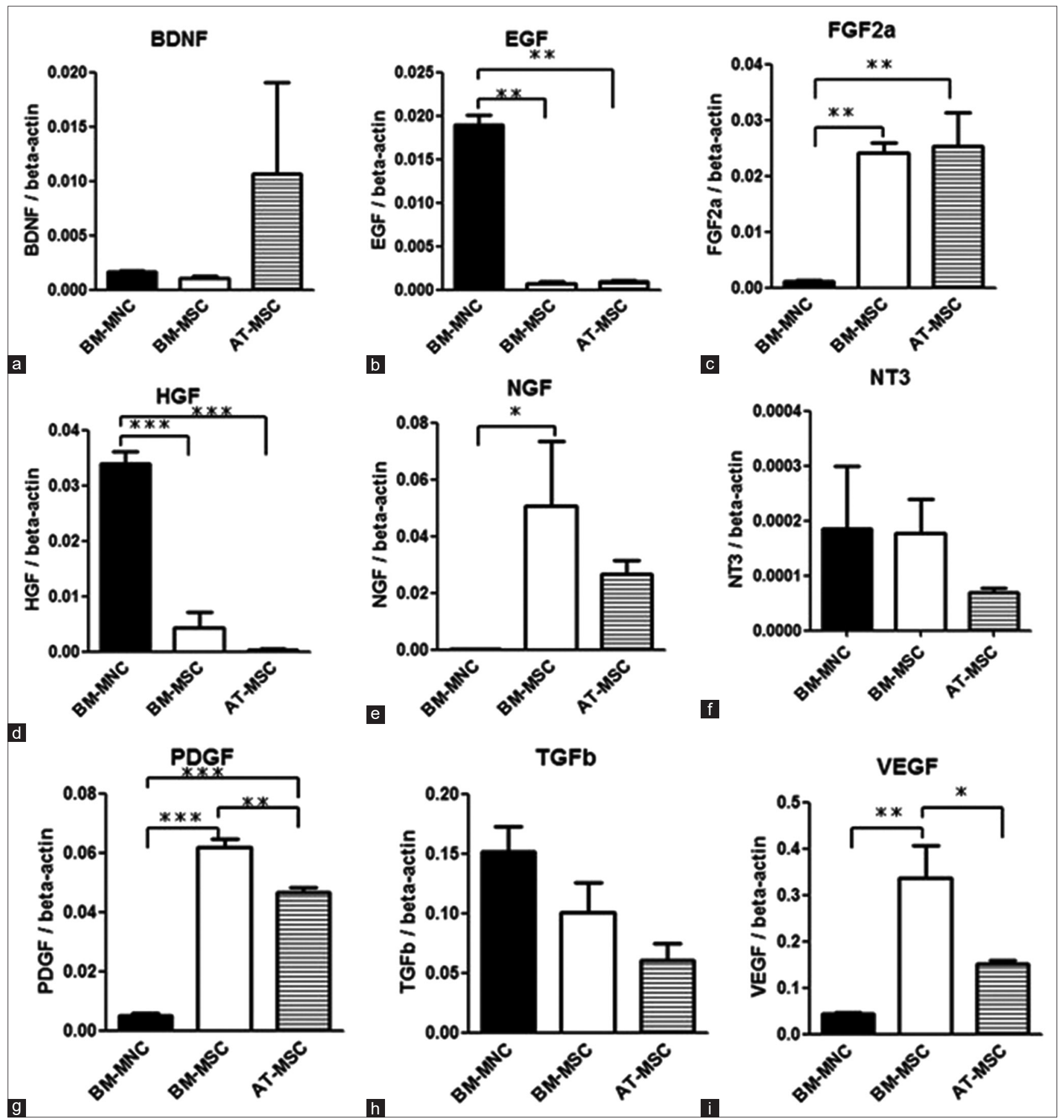

Figure-2: Quantitative real-time polymerase chain reaction. The levels of expression of dog growth factors mRNAs compared with those of bone marrow-derived mononuclear cells, adipose-derived-mesenchymal stem cells, and bone marrow-derived mesenchymal stem cells. Each value was normalized to beta-actin expression. Statistical comparisons were made with one-way analysis of variance (ANOVA) $(* p<0.05)(* * p<0.01)(* * * p<0.001)$. Means \pm SE, $n=3$. 
and AT-MSCs was 12.4 fold and 1.3 fold higher, respectively, than that in BM-MNCs and VEGF-A expression in BM-MSCs and AT-MSCs was 7.7 fold and 2.2 fold higher, respectively, than that in BM-MNCs. BDNF, NT-3, and TGF- $\beta 1$ were not significantly different between BM-MNCs and BM-and AT-MSCs. Quantitative measurements were performed by establishing a linear amplification curve from serial dilutions of plasmid DNA containing each cDNA. Each value was normalized to the expression levels of beta-actin.

\section{ILs and TNF- $\alpha$ mRNA expression}

The mRNA levels of expression of ILs and TNF- $\alpha$ in BM-MNCs, BM-MSCs, and AT-MSCs were similar to those of growth factors (Figure-3 and Table-4). In addition, mRNA expression levels of ILs and TNF- $\alpha$ did not show significant differences between BM-MSCs and AT-MSCs. However, the values in BM-MNCs showed a different tendency. IL-1b expression in BM-MNCs was 41.6 fold and 57.3 fold, IL-4 was 3.5 fold and 2.9 fold; IL-10 was 9.6 fold and 8.8 fold, and TNF- $\alpha$ was 12.0 fold and 8.7 fold higher than those in BM-MSCs and AT-MSCs, respectively. Quantitative measurements were performed by establishing a linear amplification curve from serial dilutions of plasmid DNA containing each cDNA. Each value was normalized to the expression levels of beta-actin.

\section{Growth factor secretion in cell culture}

Growth factor protein was measured by selecting the growth factors with the three highest levels of
mRNA expression in BM-MNCs in HGF, VEGF-A, and TGF- $\beta 1$ (Figure-4). The experimental samples of each cell in a cultured-conditioned medium that was made every 2 days were used. Results showed that the levels of expression of TGF- $\beta 1$ did not differ significantly between BM-MNCs, BM-MSCs, and AT-MSCs. However, VEGF-A levels of expression were higher in BM-MSCs than in BM-MNCs, whereas HGF levels of expression in BM-MNCs were higher than those in BM-MSCs and AT-MSCs $(\mathrm{p}<0.01)$.

\section{Measurement of the chronology of expressed growth factors in BM-MNCs}

To confirm the changes in the expression of HGF, VEGF-A, and TGF- $\beta 1$ in BM-MNCs, we analyzed the previously conditioned medium (Figure-5). Samples were collected every 2 days for 14 days. The expression of TGF- $\beta 1$ did not differ significantly between the start point (Day 2) and the endpoint (Day 14). However, a significant difference between the start point and the endpoint was found for the expression of VEGF-A and HGF: The expression of VEGF-A at the start point was very low, but it gradually increased to a stable level by 6-8 days. On the other hand, the expression of HGH at the start point was very high, but it gradually decreased to a stable level by 6-8 days.

\section{Evaluation of cell surface markers and differentia- tion potentials}

This study was performed using cells that have been previously evaluated for the following cell

Table-3: Growth factors expression on canine BM-MNC, BM-MSC, and AD-MSC.

\begin{tabular}{lccc}
\hline & BM-MNC & BM-MSC & AD-MSC \\
\hline BDNF & $1.6 \times 10^{-3} \pm 0.11 \times 10^{-3}$ & $1.0 \times 10^{-3} \pm 0.27 \times 10^{-3}$ & $10.0 \times 10^{-3} \pm 8.4 \times 10^{-3}$ \\
EGF & $190 \times 10^{-4} \pm 11 \times 10^{-4}$ & $6.8 \times 10^{-4} \pm 2.9 \times 10^{-4}$ & $9.7 \times 10^{-4} \pm 1.5 \times 10^{-4}$ \\
FGF2a & $1.0 \times 10^{-3} \pm 0.29 \times 10^{-3}$ & $24 \times 10^{-3} \pm 1.7 \times 10^{-3}$ & $25 \times 10^{-3} \pm 6 \times 10^{-3}$ \\
HGF & $340 \times 10^{-4} \pm 22 \times 10^{-4}$ & $42 \times 10^{-4} \pm 29 \times 10^{-4}$ & $3.9 \times 10^{-4} \pm 1.6 \times 10^{-4}$ \\
NGF & $0.2 \times 10^{-3} \pm 0.072 \times 10^{-3}$ & $51 \times 10^{-3} \pm 23 \times 10^{-3}$ & $27 \times 10^{-3} \pm 0.49 \times 10^{-3}$ \\
NT3 & $1.9 \times 10^{-4} \pm 1.1 \times 10^{-4}$ & $1.8 \times 10^{-4} \pm 0.61 \times 10^{-4}$ & $0.7 \times 10^{-4} \pm 0.07 \times 10^{-4}$ \\
PDGF-C & $0.5 \times 10^{-2} \pm 0.1 \times 10^{-2}$ & $6.2 \times 10^{-2} \pm 0.29 \times 10^{-2}$ & $4.7 \times 10^{-2} \pm 0.18 \times 10^{-2}$ \\
TGF-b1 & $1.5 \times 10^{-1} \pm 0.21 \times 10^{-1}$ & $1.0 \times 10^{-1} \pm 0.25 \times 10^{-1}$ & $0.61 \times 10^{-1} \pm 0.13 \times 10^{-1}$ \\
VEGF-A & $0.43 \times 10^{-1} \pm 0.025 \times 10^{-1}$ & $3.4 \times 10^{-1} \pm 0.7 \times 10^{-1}$ & $1.5 \times 10^{-1} \pm 0.082 \times 10^{-1}$ \\
\hline
\end{tabular}

$\mathrm{BM}-\mathrm{MNC}=$ Bone marrow-derived mononuclear cells, $\mathrm{BM}-\mathrm{MSC}=$ Bone marrow-derived mesenchymal stem cells, AD-MSC=Adipose-derived-mesenchymal stem cells, BDNF=Brain derived neurotrophic factor, VEGF-A=Vascular endothelial growth factor-a, TGF-b1=Transforming growth factor- $\beta$, PDGF-C=Platelet derived growth factor-C, NT3=Neurotrophin 3, HGF=Hepatocyte grows factor, NGF=Nerve growth factor, FGF2a=Fibroblast growth factor, $\mathrm{BDNF}=$ Brain-derived neurotrophic factor, $\mathrm{EGF}=$ Epidermal growth factor

Table-4: Interleukins expression on canine BM-MNC, BM-MSC, and AD-MSC

\begin{tabular}{lccc}
\hline & BM-MNC & BM-MSC & AD-MSC \\
\hline IL-1a & $7.4 \times 10^{-4} \pm 2.0 \times 10^{-4}$ & $2.3 \times 10^{-4} \pm 0.52 \times 10^{-4}$ & $4.3 \times 10^{-4} \pm 1.6 \times 10^{-4}$ \\
IL-1b & $35 \times 10^{-3} \pm 9.0 \times 10^{-3}$ & $0.84 \times 10^{-3} \pm 0.14 \times 10^{-3}$ & $0.6 \times 10^{-3} \pm 0.16 \times 10^{-3}$ \\
IL-4 & $1.1 \times 10^{-3} \pm 0.28 \times 10^{-3}$ & $0.32 \times 10^{-3} \pm 0.045 \times 10^{-3}$ & $0.38 \times 10^{-3} \pm 0.044 \times 10^{-3}$ \\
IL-6 & $0.24 \times 10^{-3} \pm 0.13 \times 10^{-3}$ & $2.8 \times 10^{-3} \pm 1.5 \times 10^{-3}$ & $0.43 \times 10^{-3} \pm 0.049 \times 10^{-3}$ \\
IL-10 & $3.6 \times 10^{-3} \pm 1.3 \times 10^{-3}$ & $0.38 \times 10^{-3} \pm 0.095 \times 10^{-3}$ & $0.4 \times 10^{-3} \pm 0.049 \times 10^{-3}$ \\
IL-11 & $0.36 \times 10^{-3} \pm 0.064 \times 10^{-3}$ & $4.5 \times 10^{-3} \pm 1.2 \times 10^{-3}$ & $2.6 \times 10^{-3} \pm 1.4 \times 10^{-3}$ \\
IL-17A & $5.5 \times 10^{-4} \pm 1.3 \times 10^{-4}$ & $5.6 \times 10^{-4} \pm 2.3 \times 10^{-4}$ & $4.0 \times 10^{-4} \pm 0.12 \times 10^{-4}$ \\
TNF- $\alpha$ & $4.7 \times 10^{-3} \pm 1.0 \times 10^{-3}$ & $0.4 \times 10^{-3} \pm 0.057 \times 10^{-3}$ & $0.54 \times 10^{-3} \pm 0.032 \times 10^{-3}$ \\
\hline
\end{tabular}

$\mathrm{BM}-\mathrm{MNC}=$ Bone marrow-derived mononuclear cells, $\mathrm{BM}-\mathrm{MSC}=$ Bone marrow-derived mesenchymal stem cells, AD-MSC=Adipose-derived-mesenchymal stem cells, BDNF=Brain-derived neurotrophic factor, TGF-b1=Transforming growth factor- $\beta$ 


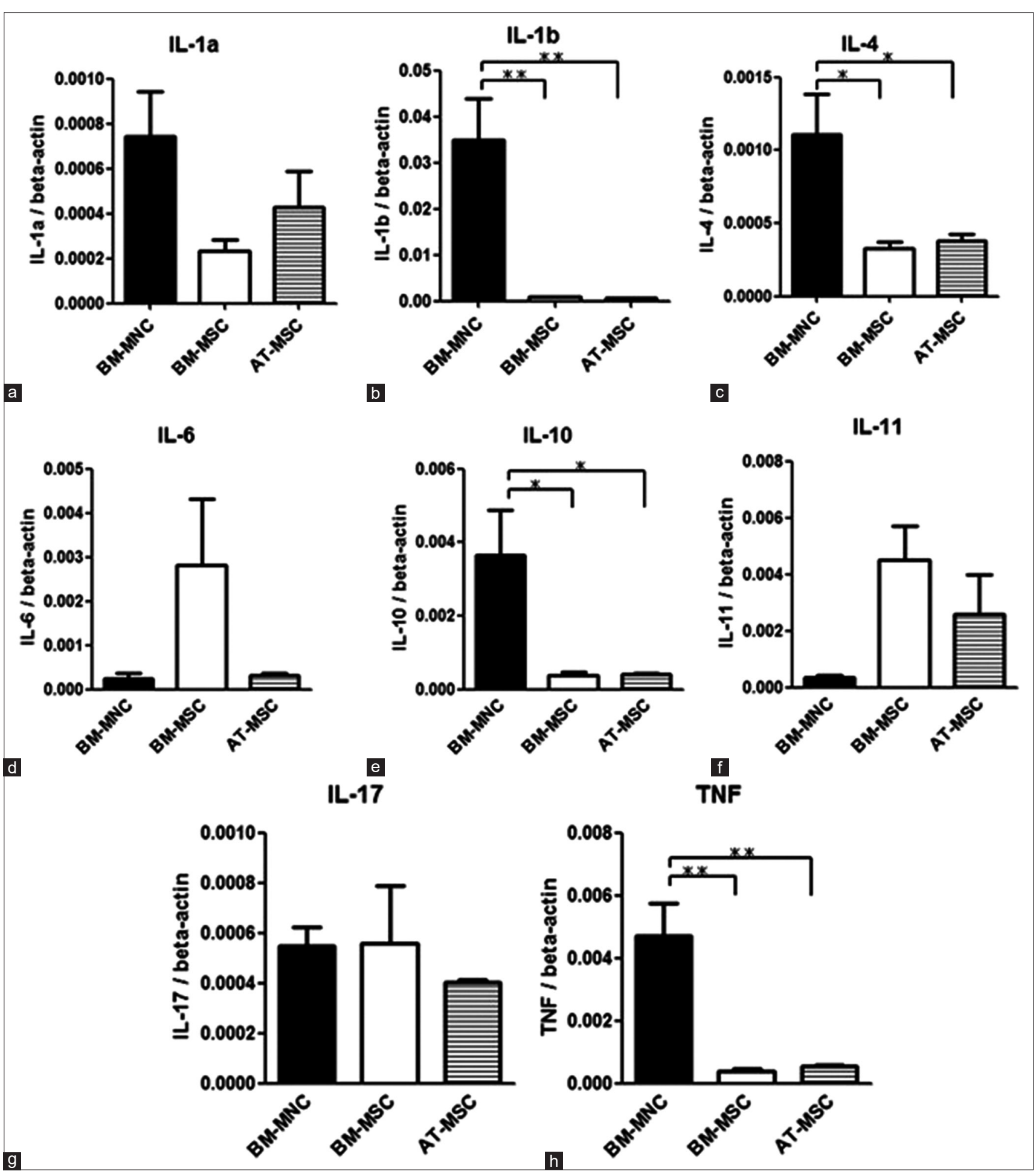

Figure-3: Quantitative real-time polymerase chain reaction. Expression levels of dog interleukin mRNAs compared with those of bone marrow-derived mononuclear cells, adipose-derived-mesenchymal stem cells, and bone marrow-derived mesenchymal stem cells. Each value was normalized to beta-actin expression. Statistical comparisons were made with one-way ANOVA $(* \mathrm{p}<0.05)(* * \mathrm{p}<0.01)(* * * \mathrm{p}<0.001)$. Means $\pm \mathrm{SE}, \mathrm{n}=3$.

surface markers [29]: CD29 (BM-MNC: $10.67 \pm 1.38 \%$; BM-MSC: $98.41 \pm 0.53 \%$; AT-MSC: $97.85 \pm 0.94 \%$ ), CD34 (BM-MNC: $8.33 \pm 0.46 \%$; BM-MSC: $0.88 \pm 0.21 \%$; AT-MSC: $0.25 \pm 0.06 \%$ ), and CD90 (BM-MNC: $25.17 \pm 0.18 \%$; BM-MSC: $19.1 \pm 2.1 \%$; AT-MSC: $22.55 \pm 2.8 \%)$. The differentiation potential of BM-MSCs and AT-MSCs has been confirmed previously.

\section{Discussion}

In this study, no significant difference in the mRNA expression and secretion of cytokines and ILs between BM-MSCs and AD-MSCs was observed. However, higher expression levels of EGF, HGF, IL-1b, IL-4, IL-10, and TNF- $\alpha$ in BM-MNCs were observed in comparison with those in MSCs. In addition, the levels of expression of FGF-2a, NGF, PDGF-C, and 


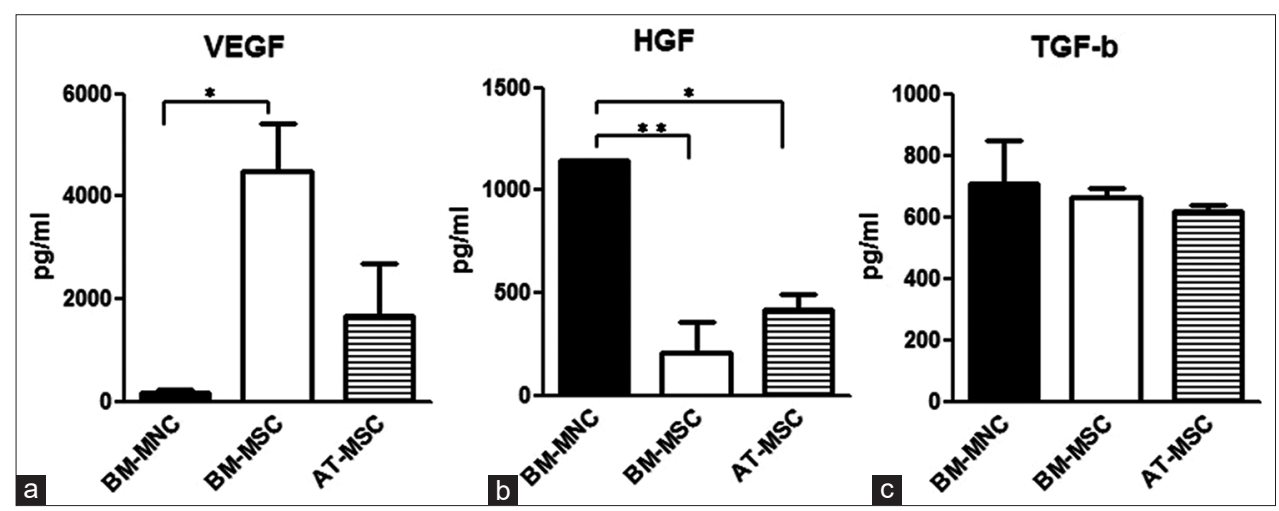

Figure-4: Evaluation of protein expression. The expression levels of vascular endothelial growth factor (VEGF), hepatocyte growth factor (HGF), and transforming growth factor (TGF)- $\beta 1$ protein were evaluated by enzyme-linked immunosorbent assays (ELISA). Comparison of each protein expression level among bone marrow-derived mononuclear cells, adiposederived-mesenchymal stem cells, and bone marrow-derived mesenchymal stem cells cultured conditioned medium. Statistical comparisons were made with one-way ANOVA $(* p<0.05)(* * p<0.01)$. Means $\pm S E, n=3$.

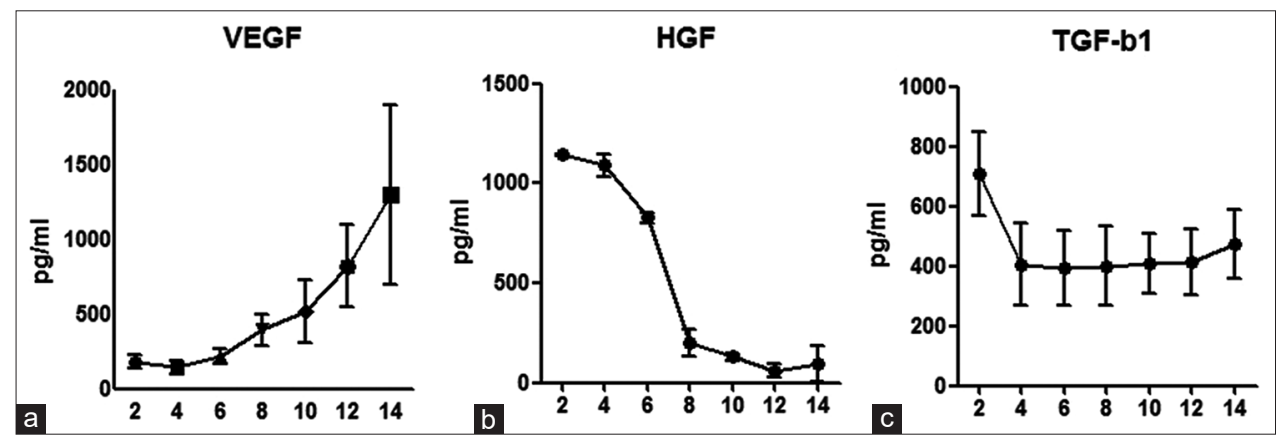

Figure-5: Evaluation of the chronology of the expressed growth factors in bone marrow-derived mononuclear cells. The expression levels of vascular endothelial growth factor-a (VEGF-A), hepatocyte grows factor, and transforming growth factor- $\beta 1$ protein were evaluated by ELISA. Samples were collected every 2 days for 14 days. Means \pm SE.

VEGF-A were also higher. BM-MNCs were found to be lower than those of MSCs. Thus, the change of BM cell character by culturing was confirmed. Moreover, TGF- $\beta 1$, which is a pleiotropic cytokine that regulates extracellular matrix production, cell proliferation, and differentiation, showed high levels of expression in all three cells that secreted cytokines.

Moreover, cytokines with high expression in BM-MNCs, such as EGF, HGF, and IL-10, have been predicted to be effective in the anti-inflammatory response and tissue regeneration [30-32]. EGF has the ability to activate cell division through the promotion of mitosis [32]. IL-10's primary role is an anti-inflammatory effect that occurs by inhibiting the differentiation of inflammatory cells and the secretion of inflammatory cytokines [31]. In particular, HGF is expected to be effective in the treatment of various diseases. Studies showed that HGF has an anti-apoptotic ability in cells and has a regenerative and protective ability in various tissues [33-35]. In addition, a study revealed HGF to have anti-fibrotic and anti-inflammatory effects [36]. These effects may be due to BM-MNC transplanted [17]. On the other hand, IL-1d and TNF- $\alpha$ are known inflammatory cytokines to have high expression in BM-MNCs $[25,36]$. Therefore, cell transplantation might lead to exacerbation of temporary inflammation. Furthermore, this runs the risk of worsening symptoms, depending on the purpose of the treatment. BM-MNCs are expected to have cell proliferation, anti-apoptosis, and anti-fibrotic effects.

In contrast, AT-MSC and BM-MSC cytokine expression, such as NGF, PDGF-C, VEGF-A, and FGF-2a, was higher than that in BM-MNCs. NGF has the ability to repair neural tissue with wounds [37], whereas PDGF-C promotes cellular proliferation and inhibits apoptosis [20]. VEGF-A constitutes a family of regulatory peptides that are capable of controlling blood vessel formation and permeability [38]. FGF-2a has been related to the differentiation control of various organs, such as the heart, liver, pancreas, and bones [39]. Moreover, FGF-2a is related to angiogenesis with PDGF-C and VEGF-A. In particular, the interaction of FGF-2a and VEGF-A contributes stable angiogenesis [40]. For this reason, AT-MSCs and BM-MSCs have been considered to contribute to cell proliferation and neural repair and angiogenesis.

Our study revealed the mRNA and protein expression profiles of canine BM-MNCs, BM-MSCs, and AT-MSCs, showing BM-MSCs and AT-MSCs to have similar expression profiles. In contrast, BM-MNCs showed unique expression profiles for HGF and EGF. The three types of cells showed a similar expression of TGF- $\beta 1$. The secretion ability of MNCs might be 
appropriate at early stages of inflammation during transplantation due to its anti-apoptotic effect. On the other hand, the secretion ability of MSCs might be appropriate during transplantation, that is, in tissue repair period, due to its cell proliferation and angiogenesis effect. However, this study only used three subjects and tendencies may be different depending on the number of samples of BDNF, NGF, IL-6, and IL-10.

\section{Conclusion}

Changes in chronologies secretion ability of growth factors seems to reflect the results of mRNA expression. TGF- $\beta 1$ secretion did not differ significantly between BM-MSC and MNC functions. In contrast, HGF secretion was decreased by time-dependent and VEGF secretion was increased by time-dependent. This result suggests that the transplantation effects obtained were different. BM-MNC was shown to have a different ability to secrete cytokines than BM-MSC and AT-MSC. And BM-MNCs were easy to handle because it does not require culturing. These suggest that BM-MNC may be applicable to various disease treatments. We found the protein and mRNA expression of cytokines that may help for tissue repair and protection, but it remains unclear whether the activity level that is required for the repair and protection of each tissue is obtained in practice. Further studies of in vivo and coculture experiments are required.

\section{Authors' Contributions}

NM and KT designed the study, drafted the manuscript, and analyzed data. FE collected samples and did ELISA analysis. HT contributed to the study design and helped in editing and revision of the manuscript. All authors read and approved the final manuscript

\section{Acknowledgments}

The authors are grateful to the Department of Comparative Animal Science, Kurashiki University of Science and The Arts, Japan, for providing funding and intellectual support to this study. The authors would like to thank Enago (www.enago.jp) for the English language review.

\section{Competing Interests} interests.

The authors declare that they have no competing

\section{Publisher's Note}

Veterinary World remains neutral with regard to jurisdictional claims in published institutional affiliation.

\section{References}

1. Caplan, A.I. (1991) Mesenchymal stem cells. J. Orthop. Res., 9(5): 641-650.

2. Huang, J.I., Beanes, S.R., Zhu, M., Lorenz, H.P., Hedrick, M.H. and Benhaim, P. (2002) Rat extra-medullary adipose tissue as a source of osteochondrogenic progenitor cells. Plast. Reconstr. Surg., 109(3): 1033-1041.

3. Liu, Z., Screven, R., Boxer, L., Myers, M.J. and Devireddy, L.R. (2018) Characterization of canine adipose-derived mesenchymal stromal/stem cells in serumfree medium. Tissue Eng. Part C Methods, 24(7): 399-411.

4. Jiang, Y., Vaessen, B. and Lenvik, T. (2002) Multipotent progenitor cells can be isolated from postnatal murine bone marrow, muscle, and brain. Exp. Hematol., 30(8): 896-904.

5. Ding, D.C., Chang, Y.H., Shyu, W.C. and Lin, S.Z. (2015) Human umbilical cord mesenchymal stem cells: A new era for stem cell therapy. Cell Transplant., 24(3): 339-347.

6. Chen, Q., Shou, P., Zheng, C., Jiang, M., Cao, G., Yang, Q., Cao, J., Xie, N., Velletri, T., Zhang, X., Xu, C., Zhang, L., Yang, H., Hou, J., Wang, Y. and Shi, Y. (2016) Fate decision of mesenchymal stem cells: Adipocytes or osteoblasts? Cell Death Differ., 23(7): 1128-1139.

7. Borghesi, J., Lima, M.F., Mario, L.C., de Almeida da Anunciação, A.R., Rabelo, A.C.S., da Silva M.G.K., Fernandes, F.A., Miglino, M.A., Carreira, A.C. and Favaron, P.O. (2019) Oliveira canine amniotic membrane mesenchymal stromal/stem cells: Isolation, characterization and differentiation. Tissue Cell, 58: 99-106.

8. Han, Y., Li, X., Zhang, Y., Han, Y., Chang, F. and Ding, J. (2019) Mesenchymal stem cells for regenerative medicine. Cells, 8(8): 886.

9. Sun, Y., Jin, K., Xie, L., Childs, J., Mao, X.O., Logvinova, A. and Greenberg, D.A. (2003) VEGF-induced neuroprotection, neurogenesis, and angiogenesis after focal cerebral ischemia. J. Clin. Investig., 111(12): 1843-1851.

10. Abughanam, G., Elkashty, O.A., Liu, Y., Bakkar, M.O. and Tran, S.D. (2019) Mesenchymal stem cells extract (MSCsE)-based therapy alleviates xerostomia and keratoconjunctivitis sicca in Sjogren's syndrome-like disease. Int. J. Mol. Sci., 20(19): 4750.

11. Seo, K.W., Lee, H.W., Oh, Y.I., Ahn, J.O., Koh, Y.R., Oh, S.H., Kang, S.K. and Youn, H.Y. (2011) Anti-tumor effects of canine adipose tissue-derived mesenchymal stromal cell-based interferon- $\beta$ gene therapy and cisplatin in a mouse melanoma model. Cytotherapy, 13(8): 944-955.

12. Lu, X., Wang, X., Nian, H., Yang, D. and Wei, R. (2017) Mesenchymal stem cells for treating autoimmune dacryoadenitis. Stem Cell Res. Ther., 8(1): 126.

13. Sun, C.K., Zhen, Y.Y., Leu, S., Tsai, T.H., Chang, L.T., Sheu, J.J., Chen, Y.L., Chua, S., Chai, H.T., Lu, H.I., Chang, H.W., Lee, F.Y. and Yip, H.K. (2014) Direct implantation versus platelet-rich fibrin-embedded adipose-derived mesenchymal stem cells in treating rat acute myocardial infarction. Int. J. Cardiol., 173(3): 410-423.

14. Zeira, O., Asiag, N., Aralla, M., Ghezzi, E., Pettinari, L., Martinelli, L., Zahirpour, D., Dumas, M.P., Lupi, D., Scaccia, S., Konar, M. and Cantile, C. (2015) Adult autologous mesenchymal stem cells for the treatment of suspected non-infectious inflammatory diseases of the canine central nervous system: Safety, feasibility and preliminary clinical findings. J. Neuroinflammation, 12: 181.

15. Trigg, M.E. (1988) Bone marrow transplantation for treatment of leukemia in children. Pediatr. Clin. North Am., 35(4): 933-948.

16. Henrich, D., Seebach, C., Verboket, R., Schaible, A., Marzi, I. and Bonig, H. (2018) The osteo-inductive activity of bone-marrow-derived mononuclear cells resides within the CD14+ population and is independent of the CD34+ population. Eur. Cell Mater., 35: 165-177.

17. Tamura, K., Harada, Y., Nagashima, N., Itoi, T., Ishino, H., Yogo, T., Nezu, Y., Hara, Y., Suzuki, Y., Ide, C. and Tagawa, M. (2012) Autotransplanting of bone marrow-derived mononuclear cells for complete cases of canine paraplegia and loss of pain perception, secondary to intervertebral disc herniation. Exp. Clin. Transplant., 10(3): 263-272.

18. Björkholm, C. and Monteggia, L.M. (2016) BDNF a key transducer of antidepressant effects. Neuropharmacology, 
102: 72-79.

19. Bracci-Laudiero, L. and De Stefano, M.E. (2016) NGF in early embryogenesis, differentiation, and pathology in the nervous and immune systems. Curr. Top. Behav. Neurosci., 29: $125-152$.

20. Tamama, T., Fan, H., Griffith, G., Blair, H. and Wells, A. (2006) Epidermal growth factor as a candidate for ex vivo expansion of bone marrow-derived mesenchymal stem cells. Stem Cells, 24(3): 686-695.

21. Ng, F., Boucher, S. and Koh, S. (2008) PDGF-C, TGF- $\beta$, and FGF signaling is important for differentiation and growth of mesenchymal stem cells (MSCs): Transcriptional profiling can identify markers and signaling pathways important in differentiation of MSCs into adipogenic, chondrogenic, and osteogenic lineages. Blood, 112(2): 295-307.

22. Zhu, X.Y., Zhang, X.Z., Xu, L., Zhong, X.Y., Ding, Q. and Chen, Y.X. (2009) Transplantation of adipose-derived stem cells overexpressing hHGF into cardiac tissue. Biochem. Biophys. Res. Commun., 379(4): 1084-1090.

23. Zheng, L., Zhao, C., Du, Y., Lin, X., Jiang, Y., Lee, C., Tian, G., Mi, J., Li, X., Chen, Q., Ye, Z., Huang, L., Wang, S., Ren, X., Xing, L., Chen, W., Huang, D., Gao, Z., Zhang, S., Lu, W., Tang, Z., Wang, B., Ju, R. and Li, X. (2016) PDGF-CC underlies resistance to VEGF-A inhibition and combinatorial targeting of both suppresses pathological angiogenesis more efficiently. Oncotarget, 7(47): 77902-77915.

24. Xu, F., Liu, Y., Shi, L., Cai, H., Liu, W., Hu, Y., Li, Y. and Yuan, W. (2017) RGS3 inhibits TGF- $\beta 1 /$ Smad signalling in adventitial fibroblasts. Cell Biochem. Funct., 35(6): 334-338.

25. Wooff, Y., Man, S.M., Aggio-Bruce, R., Natoli, R. and Fernando, N. (2019) IL-1 family members mediate cell death, inflammation and angiogenesis in retinal degenerative diseases. Front. Immunol., 10: 1618.

26. Paul, S.R., Bennett, F., Calvetti, J.A., Kelleher, K., Wood, C.R., O'Hara, R.M. Jr., Leary, A.C., Sibley, B., Clark, S.C. and Williams, D.A. (1990) Molecular cloning of a cDNA encoding interleukin 11, a stromal cell-derived lymphopoietic and hematopoietic cytokine. Proc. Natl. Acad. Sci. U. S. A., 87(19): 7512-7516.

27. Kuwabara, T., Ishikawa, F., Kondo, M. and Kakiuchi, T. (2017) The role of IL-17 and related cytokines in inflammatory autoimmune diseases. Mediators Inflamm., 2017: 3908061.

28. Ghoreschi, K., Thomas, P., Breit, S., Dugas, M., Mailhammer, R., van Eden, W., van der Zee, R., Biedermann, T., Prinz, J., Mack, M., Mrowietz, U., Christophers, E., Schlöndorff, D., Plewig, G., Sander, C.A. and Röcken, M. (2003) Interleukin-4 therapy of psoriasis induces Th2 responses and improves human autoimmune disease. Nat. Med., 9(1): 40-46.

29. Takemitsu, H., Zhao, D., Yamamoto, I., Harada, Y., Michishita, M. and Arai, T. (2012) Comparison of bone marrow and adipose tissue-derived canine mesenchymal stem cells. BMC Vet. Res., 8: 150.

30. Kinnaird, T., Stabile, E., Burnett, M.S. and Epstein, S.E. (2004) Bone-marrow-derived cells for enhancing collateral development: Mechanisms, animal data, and initial clinical experiences. Circ. Res., 95(4): 354-363.

31. Franchimont, D., Martens, H., Hagelstein, T., Louis, E., Dewe, W., Chrousos, P., Belaiche, J. and Geenen, V. (1999) Tumor necrosis factor-alpha decreases, and interleukin-10 increases, the sensitivity of human monocytes to dexamethasone: Potential regulation of the glucocorticoid receptor. J. Clin. Endocr. Metab., 84(8): 2834-2839.

32. Sassone-Corsi, P., Mizzen, C.A., Cheung, P., Crosio, C., Monaco, L., Jacquot, S., Hanauer, A. and Allis, C.D. (1999) Requirement of Rsk-2 for epidermal growth factor-activated phosphorylation of histone H3. Science, 285(5429): 886-891.

33. Motoi, S., Toyoda, H., Obara, T., Ohta, E., Arita, Y., Negishi, K., Moriya, K., Kuboi, Y., Soejima, M., Imai, T., Ido, A., Tsubouchi, H. and Kawano, T. (2019) Anti-apoptotic effects of recombinant human hepatocyte growth factor on hepatocytes were associated with intrahepatic hemorrhage suppression indicated by the preservation of prothrombin time. Int. J. Mol. Sci., 20(8): 1821.

34. Oliveira, A.G., Araújo, T.G., Carvalho, B.M., Rocha, G.Z., Santos, A. and Saad, M.J.A. (2018) The role of hepatocyte growth factor (HGF) in insulin resistance and diabetes. Front. Endocrinol. (Lausanne), 9: 503.

35. Matsumoto, K., Funakoshi, H., Takahashi, H. and Sakai, K. (2014) HGF-met pathway in regeneration and drug discovery. Biomedicines, 2(4): 275-300.

36. Wu, X., Xu, W., Feng, X., He, Y., Liu, X., Gao, Y., Yang, S., Shao, Z., Yang, C. and Ye, Z. (2015) TNF-a mediated inflammatory macrophage polarization contributes to the pathogenesis of steroid-induced osteonecrosis in mice. Int. J. Immunopathol. Pharmacol., 28(3): 351-361.

37. Ramer, M.S., Priestley, J.V. and McMahon, S.B. (2000) Functional regeneration of sensory axons into the adult spinal cord. Nature, 403(6767): 312-316.

38. LeCouter, J., Moritz, D.R., Li, B., Phillips, G.L., Liang, X.H., Gerber, H.P., Hillan, K.J. and Ferrara, N. (2003) Angiogenesis-independent endothelial protection of liver: Role of VEGFR-1. Science, 299(5608): 890-893.

39. Spence, J.R., Mayhew, C.N., Rankin, S.A., Kuhar, M.F., Vallance, J.E., Tolle, K., Hoskins, E.E., Kalinichenko, V.V., Wells, S.I., Zorn, A.M., Shroyer, N.F. and Wells, J.M. (2011) Directed differentiation of human pluripotent stem cells into intestinal tissue in vitro. Nature, 470(7332): 105-109.

40. Cao, R., Bråkenhielm, E., Pawliuk, R., Wariaro, D., Post, M.J., Wahlberg, E., Leboulch, P. and Cao, Y. (2003) Angiogenic synergism, vascular stability and improvement of hind-limb ischemia by a combination of PDGF-BB and FGF-2. Nat. Med., 9(5): 604-613. 